\section{GET HELP DRIVING NEW PATIENTS TO YOUR PRACTICE}

Breeze Digital's recently launched SEO Dental Jumpstart service offers a holistic review of existing online marketing activity and a customised online marketing plan for dental practices looking to attract new patients.

A search marketing review includes observations about a website's functionality and usability, assessing its position in the natural search rankings for a range of targeted search terms important to the dental practice owner. For example, a practice may want to be found online for its tooth whitening or dental implants services, and its website is not working hard enough to be discovered for these terms on search engines such as Google.

The experts at Breeze Digital discuss overall aims

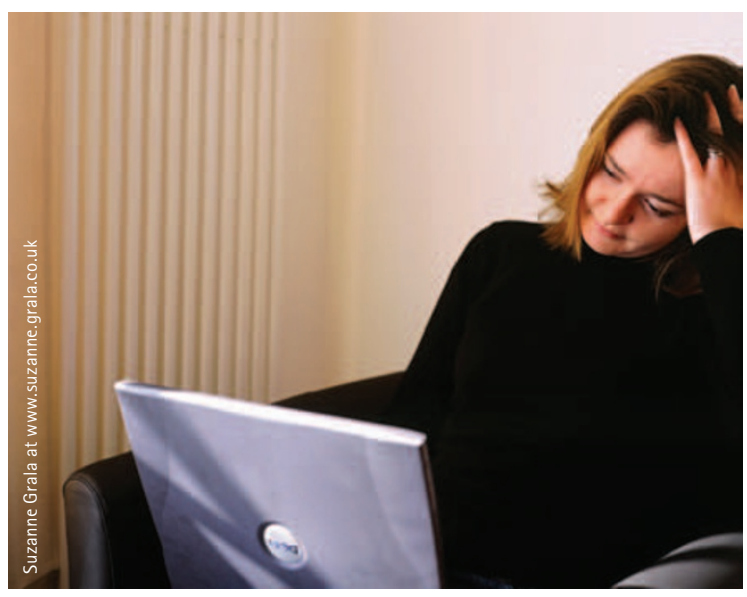

and objectives with clients, and start by generating a benchmark report using dedicated software, which highlights weak areas of website content. Next, a customised online marketing plan is developed which may recommend using one or more online marketing tools - such as a customised paid search marketing campaign (PPC) on Google AdWords, or a search engine optimisation (SEO) programme of work, which could include new 'tag' creation or 'content optimisation'.

If you're about to invest in a website, or want to improve your existing one - or even if you don't have a website online marketing can offer a flexible and accountable way to help drive new leads to your dental practice.

Call Breeze Digital on 07952 608169 or 07546441237 for information (please mention the $B D J$ ) or visit www. breezedigital.co.uk.
INTRA-ORAL SOPHISTICATION

Durr Dental has taken a simple technology concept and refined it to its maximum potential. The intra-oral camera has provided both dentists and their patients with a revealing window into the realm of dental disease, conferring greater transparency and more lucid communication. Durr has consolidated a range of technologies to ensure this invaluable tool has its most sophisticated application yet.

One of the most versatile aspects of Durr Dental's VistaCam iX is the interchangeable head mechanism. Four heads are available, one providing high resolution images of the oral environment; a macro head for close-ups of up to 100× zoom; a proof head for caries diagnosis with a colour-coded scale; and an LED curing light. Data transmission is fully digital through a USB port to a PC, or can function as a stand-alone version without a PC. The unit also holds the promise of additional heads being added in the future, which will further enhance its range of applications.

Durr has also set a benchmark in its design, winning the prestigious Red Dot Design Award in the process. The ergonomic head rotates a full $360^{\circ}$ to ensure that every part of the oral cavity is easily accessible, and a motion sensor automatically switches the camera on and off to ensure efficient usage. Its smooth finish enables easy disinfection and sheathing for cross infection.

For more information call 01536526740. 